\section{Pleuropulmonary blastoma in a 3-year-old male child with recurrence in less than two months time}

Reyaz Ahmad Lone, ${ }^{1}$ Mohd Lateef Wani, ${ }^{1}$ Shadab Nabi Wani, ${ }^{1}$ AbdulGani Ahangar, ${ }^{1}$ Furqan Nizami, ${ }^{1}$ Zahur Hussain, ${ }^{1}$

Mubasher Ahmad Bhat, ${ }^{2}$

Farooq Ahmad Ganie, ${ }^{1}$

Nasir-ud-din Wani ${ }^{1}$

Departments of ${ }^{1}$ Cardiovascular and

Thoracic Surgery and ${ }^{2}$ Anaesthesia,

Sher-i-Kashmir Institute of Medical

Sciences, Srinagar, India

\section{Abstract}

Pleuropulmonary blastoma is a rare childhood neoplasm accounting for less than one percent of all primary malignant lung tumours of children less than six years of age. Metastasis to central nervous system, orbit and iris, bone, contralateral lung and rarely adrenalglands, liver, kidney and pancreas has been described. We present a case of pleuropulmonary blastoma in a 3-year-old child who had a recurrence in less than three months, after surgical resection.

\section{Introduction}

Pleuropulmonary blastoma (PPB) is a rare dysembryonic neoplasm. It was first described by Manivel et al. in 1988 in eleven children. ${ }^{1}$ It is a dysembryonic neoplasm of the thoraco-pulmonary mesenchyme and arises from the lung, pleural surface or both. ${ }^{2}$ The exact histogenesis is unknown. Pleuropulmonary blastoma is classified into: type I (cystic), type II (cystic and solid) and type III (predominantly solid) lesions. ${ }^{3}$ Metastasis to the cervical lymph nodes are very rare. ${ }^{4}$ Do to the rarity of this disease, pathogenesis and therapeutic management of this condition remain controversial. Here we report a case of pleuropulmonary blastoma in a 3-year-old child with a recurrence noted in less than three months after surgical resection.

\section{Case Report}

A 3-year-old male child presented with breathlessness, cough and intermittent fever for the last 2 months. He received symptomatic treatment for 4-6 weeks with no clinical improvement. A chest x-ray showed a radioopaque right hemithorax. A chest computed tomography (CT) demonstrated a large inhomogeneous mass occupying almost the entire right chest (Figure 1). A shift of the mediastinal structures was noted to the left. A bronchoscopy was not performed. Differential diagnosis included either a pleuropulmonary blastoma or a primitive neuroectodermal tumour. The patient then underwent thoracotomy. At thoractomy, a huge fleshy tumour measuring $20 \times 15 \times 5 \mathrm{~cm}$ of variegated consistency with soft and solid components arising from right lower lobe infiltrating into the pericardium, chest wall, great vessels and almost occupying the entire right side of the chest. There was no evidence of hilar or mediastinal lymphadenopathy. A right pneumonectomy was performed. On histologic examination, a biphasic cell population consisting of primitive blastomatous cells and spindle shaped sarcomatous cells were noted. These features were consistent with a diagnosis of pleuropulmonary blastoma (stage IIIA). Postoperative course was uneventful and the child was discharged after 2 weeks of hospital stay. The patient was to follow-up with the Medical Oncology Department for postoperative chemotherapy. However, the patient was from a remote area and did not follow-up. Consequently, no chemotherapy or radiotherapy was given postoperatively. The father of the patient brought him back one and a half month after discharge. At this time, the child was in severe respiratory distress, cyanosed, febrile and was having a productive cough. Oxygen inhalation, nebulization with bronchodilators;
Correspondence: Mohd Lateef Wani, Department of Cardiovascular and thoracic Surgery and Anaesthesia, Sher-i-Kashmir Institute of Medical Sciences, Srinagar, India.

E-mail: latifs_dr@yahoo.com

Key words: lung, malignant tumour, pleuro-pulmonary blastoma.

Received for publication: 24 July 2011.

Revision received: 29 March 2012.

Accepted for publication: 2 May 2012.

This work is licensed under a Creative Commons Attribution NonCommercial 3.0 License (CC BYNC 3.0).

(C) Copyright R.A. Lone et al., 2012

Licensee PAGEPress, Italy

Chest Disease Reports 2012; 2:e9

doi:10.4081/cdr.2012.e9

intravenous antibiotics and endotrachial suction was done. Chest radiograph revealed a radio opaque shadow occupying the whole right side of the chest pushing the mediastinal structures and heart towards left side. Ultrasound of the chest revealed a large heterogeneous mass almost completely replacing the right hemithorax. A CT chest was planned after initial resuscitation. Patients' blood gases were grossly deranged. In view of severe hypoxia and acidosis the child was transferred to the surgical intensive care unit for needful ventilation but the patient developed sudden cardiac arrest and could not be revived after resuscitation and was declared dead.

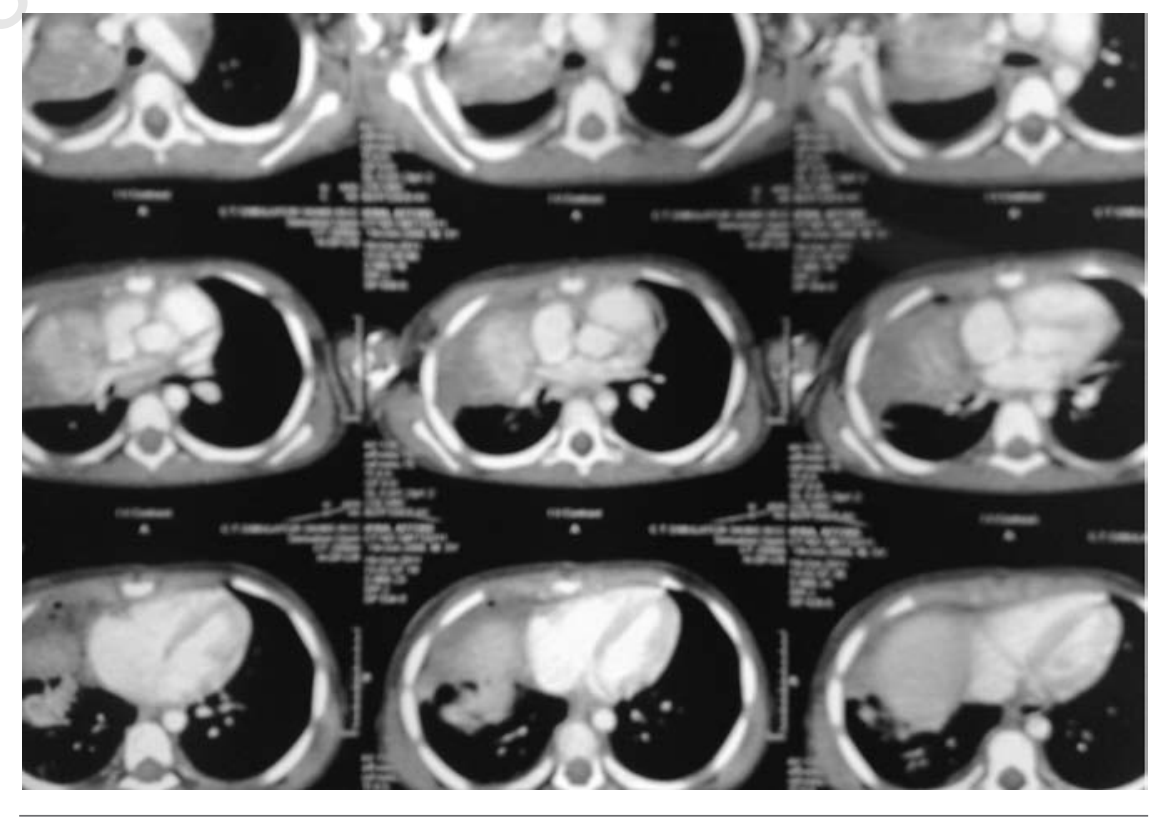

Figure 1. Chest computed tomography demonstrating a large inhomogeneous mass occupying almost the entire right chest. 


\section{Discussion}

Pleuro-pulmonary blastoma (PPB) is a rare and aggressive tumour that affects children. PPB account $1 \%$ of all primary malignant lung tumours in the pediatric population. ${ }^{1} \mathrm{PPB}$ is a disease with distinct clinicopathologic characteristics.

This neoplasm is histologically characterized by primitive blastoma and a malignant mesenchymal stroma that often demonstrates multidirectional differentiation. ${ }^{4}$

Manivel and associates coined the term PPB to describe a specific subtype of pulmonary blastoma on the basis of its exclusive clinical presentation in childhood and its pathologic features of variable anatomic location, primitive embryonic-like blastoma and stroma, absence of carcinomatous component, and potential for sarcomatous differentiation. ${ }^{2}$ Denher and associates classified PPB into three histologicalvariants: type 1 with purely cystic tumours, type 3 with predominantly solid tumours, and type 2 as an intermediate type. ${ }^{3}$ Our patient belonged to type 2. Type 1 PPB is seen in young infants where as older infants and children tends to present with type 2 and type 3 PPB which have poor prognosis and high recurrence rate. $^{5}$

A progression from type 1 to type 3 over time may occur. ${ }^{6}$ This neoplasm occurs not only in the lung, but may also arise from the mediastinum, diaphragm and/or pleura. This has raised the possibility that PPB might originate from the splanchnopleural or somatopleural mesoderm. Five year survival for type II and III lesions is approximately $42 \%$ after multimodality therapy.

Patients with pleural, mediastinal and extrapulmonary involvement have a worse prognosis. Treatment of choice for pleuropulmonary blastoma is radical surgery followed by chemotherapy and radiotherapy, because the response to radiotherapy alone is not as good. In our case, the patient did not receive any adjuvant treatment, which may have contributed to the early recurrence of the tumour.

\section{References}

1. Priest JR, Watterson J, Strong L, et al. Pleuropulmonary blastoma: a marker for familial disease. J Pediatr 1996;128:220-4.

2. Manivel JC, Priest JR,Watterson J, et al. Pleuropulmonary blastoma. The so-calledpulmonary blastoma in childhood. Cancer 1988;62:1516-26.

3. Dehner LP, Watterson J, Priest J. Pleuropulmonary blastoma. A unique intrathoracic pulmonary neoplasm of childhood. Perspect Pediatr Pathol 1995; 18:214-26.

4. Priest JR, McDermott MB, Bhatia S, et al. Pleuropulmonary blastoma: a clinicopathological study of 50 cases. Cancer 1997;80: 147-61.

5. Al-Backer N, Paliqandla PS, Su W, et al. Type 1 PPB in a 3-year-old male with a cystic lung lesion. J Pediatr Surg 2006;41:13-5.

6. Wright JR Jr. Pleuropulmonary blastoma: A case report documenting transition from type 1 (cystic) to type 3 (solid). Cancer 2000;88:2853. 